\title{
Multiphysics simulation of a microfluidic perfusion chamber for brain slice physiology
}

\author{
Hector H. Caicedo • Maximiliano Hernandez • \\ Christopher P. Fall • David T. Eddington
}

Published online: 13 May 2010

(C) The Author(s) 2010. This article is published with open access at Springerlink.com

\begin{abstract}
Understanding and optimizing fluid flows through in vitro microfluidic perfusion systems is essential in mimicking in vivo conditions for biological research. In a previous study a microfluidic brain slice device ( $\mu \mathrm{BSD})$ was developed for microscale electrophysiology investigations. The device consisted of a standard perfusion chamber bonded to a polydimethylsiloxane (PDMS) microchannel substrate. Our objective in this study is to characterize the flows through the $\mu \mathrm{BSD}$ by using multiphysics simulations of injections into a pourous matrix to identify optimal spacing of ports. Three-dimensional computational fluid dynamic (CFD) simulations are performed with CFD-ACE + software to model, simulate, and assess the transport of soluble factors through the perfusion bath, the microchannels, and a material that mimics the porosity, permeability and tortuosity of brain tissue. Additionally, experimental soluble factor transport through a brain slice is predicted by and compared to simulated fluid flow in a volume that represents a porous matrix material. The computational results are validated with fluorescent dye experiments.
\end{abstract}

Keywords Microfluidics - Computational fluid dynamics (CFD) simulations · Organotypic brain slice cultures (OBSC)

Hector H. Caicedo and Maximiliano Hernandez contributed equally to this work.

H. H. Caicedo $\cdot$ M. Hernandez $\cdot$ C. P. Fall $\cdot$ D. T. Eddington $(\bowtie)$ Department of Bioengineering, University of Illinois at Chicago, Chicago, IL, USA

e-mail: dte@uic.edu

\section{Introduction}

Microfluidic perfusion systems for thick brain slice cultures enable the control of in vitro microenvironments. Since the purpose of in vitro tissue culture is often to investigate the function of live tissue, devices must be developed to both test and keep samples alive (Blake et al. 2007). Therefore, such devices must not only allow for control over experimental parameters, but also emulate in vivo conditions (Choi et al. 2007). In both cases, the delivery of soluble factors in controlled concentrations to localized regions of a tissue sample is desirable.

A successful device for thick brain slice culture accomplishes three key tasks: (1) provides oxygen to the sample tissue to sustain neurons and supporting cells; (2) allow nutrient and waste exchange; and (3) enable perfusion, the controlled delivery of soluble factors to highly localized regions of the sample tissue (Choi et al. 2007). Several designs have been proposed to achieve tissue preservation and controlled perfusion. For example, tissue resting on a nylon mesh through which nutrients from the bathing fluid are delivered by capillary action (Haas et al. 1979), brain slices attached to a cover slip and kept at an interface of nutrient medium and air in a rollertest tube (Gahwiler 1981; Gahwiler 1988), brain slices exposed to less mechanical stress by using solenoid valves that quickly switch perfusion media (Kelso et al. 1983), and acute slices resting on top of micropillars that circulate the artificial cerebral spinal fluid (ACSF) (Passeraub et al. 2003). While tissue preservation can be achieved by immersion in a circulating and oxygenated bath of ACSF (Dingledine et al. 1980; Aghajanian and Rasmussen 1989), the delivery of soluble factors to localized brain slice regions can be achieved by means of a low velocity microflow such as passive pumping through a microfluidic 
network (Mohammed et al. 2008); by using laminar flow to deliver stimulants to specific regions (Blake et al. 2007) by using external forcing mechanisms such as micro pumps to induce flow (Berthier and Beebe 2007); by local microinjection through microfluidic probes (Neeves et al. 2006) or infusion catheters (Linninger et al. 2008) that must be positioned in very close proximity to the target tissue. In our previous work (Mohammed et al. 2008), we achieved both the perfusion of ACSF through the chamber bath and the delivery of dopamine into the channels of the $\mu \mathrm{BSD}$. Although our system was simple and allowed perfusion and site-specific stimulation of brain slices with a soluble factor, the ensuing fluid transport through the perfusion chamber and microchannels was not fully characterized. The purpose of the present study is to quantitatively characterize a $\mu \mathrm{BSD}$ for its use in controlled physiology investigations. The $\mu \mathrm{BSD}$ characterized here is a dual component assembly consisting of a standard perfusion chamber bonded to a PDMS microchannel substrate. The standard perfusion chamber is obtained from Warner Instruments, and has been specifically designed for low profile diamond bath tissue cultures. The $\mu \mathrm{BSD}$ characterized here is a device that consists of a parallel network of four discrete PDMS microfluidic channels, $250 \mu \mathrm{m}$ width, bonded to a coverglass substrate. Four channels were chosen initially to validate the design, however the layout of these could be tailored for addressing specific regions of slices depending on the application. Each microfluidic channel has a single via hole, $65 \mu \mathrm{m}$ diameter, to deliver soluble factors to an intact slice positioned directly above the microchannels. The device is docked to an off the shelf perfusion chamber and integrated to a standard electrophysiology set-up. The $2 \mathrm{~mm}$ height chamber has a diamond-shaped bath that assures a laminar flow across the bath. The distribution and number of the microchannels are only limited by the size of the brain slice (approx. $9 \mathrm{~mm} \times 5 \mathrm{~mm}$ ); it is possible to have an array of channels covering the whole slice with multiples via holes, of varied geometries, spanning different regions.

The PDMS substrate is fabricated using replica molding methods; a detailed description of the fabrication process can be found elsewhere (Mohammed et al. 2008). In operation, the $\mu \mathrm{BSD}$ allows localized brain slice stimulation by either passive or externally forced pumping. Since the microscopic scale at which perfusion occurs in this device is a key factor in controlling the brain slice microenvironment (Walker et al. 2004; Blake et al. 2007), a good understanding of the fluid mechanics and mass transfer that take place is required. Therefore, multiphysics simulations are performed with computational fluid dynamics (CFD) software (ESI CFD Research Corporation 2009) to model the transport of soluble factors through the overall set-up and assess the influences of each micro environmental parameter. Additional simulations are also carried out to predict transport of soluble factors through a material that mimics the porosity, density, and tortuosity of brain tissue. The results from the computational simulations provide insights for the improvement of current $\mu$ BSDs and development of new generation of these microfluidic devices. Currently, the $\mu \mathrm{BSD}$ is being used for electrophysiological experiments. As the device is docked to an off the shelf perfusion chamber and integrated to a standard electrophysiology set-up, brain tissue can be precisely stimulated by soluble factors delivered through the vias in the microfluidic device and the response in the tissue can be measured amperometrically. Another approach could use one via hole to deliver a liquid to a specific region of the brain tissue and sampling the response (possibly chemicals released from cells) by using other via holes distantly distributed. This allows the brain tissue to be constantly perfused by bulk solution, while individual locations can be individually or simultaneously accessed using the microapertures. Also, this gives additional information about the activity of brain region or culture. Other potential application of the uBSD that could be explored in the future, using organotypic slices, include the delivery of neurochemicals that promote either the regeneration of neurons or local injury for apoptosis studies; or the delivery of viruses for localized transfection. All of these studies require complete understanding of the fluid properties through the porous matrix of brain tissue and provides the motivation of this work.

\section{Methods}

\subsection{Simulating flows without a modeled brain slice}

The dimensions of a $\mu$ BSD set-up were recorded and a 3D model of the resulting fluid body (not including a brain slice) was generated as shown in Fig. 1(a). Detailed images or photographs of the $\mu \mathrm{BSD}$ with and without a brain slice can be found in our previous work (Mohammed et al. 2008). Since all soluble factors involved in the operation of the $\mu \mathrm{BSD}$ are present in water-based aqueous solutions at room temperature $(300 \mathrm{~K})$, the physical properties of these soluble factors were approximated in simulation by those of pure liquid water at room temperature as shown in Table 1. To model liquids that flow through the $\mu \mathrm{BSD}$, bath fluid was simulated as a $0 \mathrm{M} \mathrm{H}_{2} \mathrm{O}$ liquid entering from the chamber inlet and fluid was simulated as a $15 \mu \mathrm{M}$ Fluorescein isothiocyanate (FITC) liquid delivered into the chamber through the microchannels.

Fluid velocities at the chamber inlet were chosen to include $0.068 \mathrm{~m} / \mathrm{s}$, a fluid velocity that corresponds to a volumetric flow rate of $1.7 \mathrm{ml} / \mathrm{min}$ that is typically used for $\mu$ BSD applications (Mameli et al. 2005; Crowder et al. 
Fig. 1 Three dimensional CFD model of the fluid body in a microfluidic brain slice device ( $\mu$ BSD) showing (a) the geometry of the perfusion bath, four microchannels, inlets, and outlets, and (b) a finite element mesh of the model using tetrahedral elements. The latter model consists of the perfusion bath, one microchannel, and a disc located in the middle of the perfusion bath to mimic a brain slice sample and its material properties
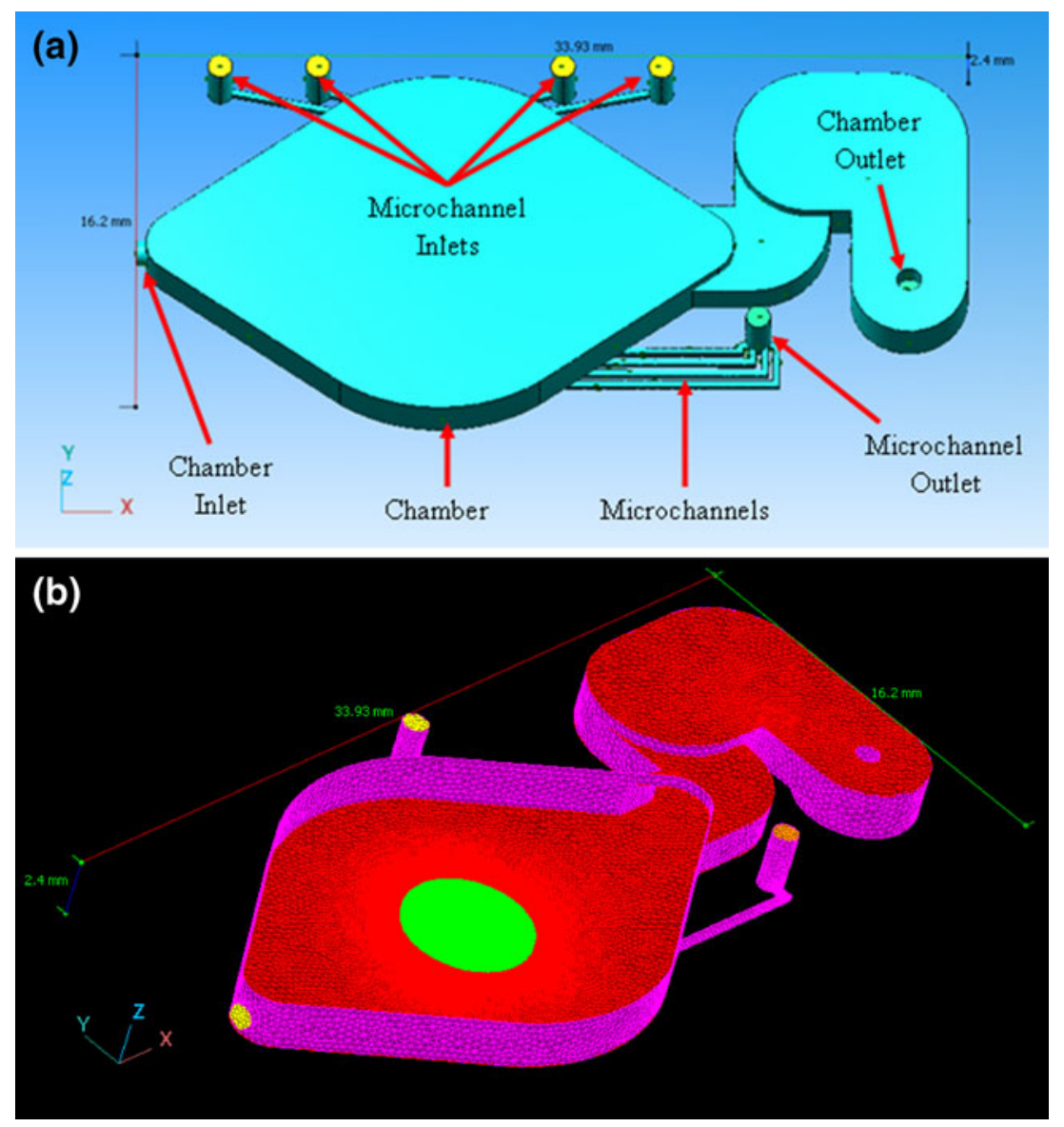

2006). The entering velocity of bath fluid at the chamber inlet was varied from $0.0125 \mathrm{~m} / \mathrm{s}$ to $0.5 \mathrm{~m} / \mathrm{s}$, corresponding to flow rates from $0.29 \mathrm{ml} / \mathrm{min}$ to $11.78 \mathrm{ml} / \mathrm{min}$. Additionally, to match experimental conditions, the entering velocity of soluble factors at the microchannel inlets was maintained constant at $0.001 \mathrm{~m} / \mathrm{s}(0.024 \mathrm{ml} / \mathrm{min})$ for all simulations.
2.2 Experimental study for assessing perfusion without brain slice

To evaluate the accuracy of CFD simulations, experiments preformed under similar conditions were carried out. In producing the experimental flow, fluorescent dye was

Table 1 Parameters used for the simulations of flow and mass transport through the $\mu$ BSD and porous media

\begin{tabular}{ll}
\hline Parameter & Value \\
\hline Room temperature & $300 \mathrm{~K}$ \\
Reference pressure (atmospheric pressure) & $10,000 \mathrm{~N} / \mathrm{m}^{2}$ \\
Density $(\rho)$ & $1,000 \mathrm{~kg} / \mathrm{m}^{3}$ \\
Viscosity $(\mu)$ & $0.001 \mathrm{~Pa} \cdot \mathrm{s}$ \\
Diffusivity $(D)$ & $1 \mathrm{E}-10 \mathrm{~m} / \mathrm{s}$ \\
Bath fluid & $0 \mathrm{M} \mathrm{H}_{2} \mathrm{O}$ \\
Soluble factor fluid & $1 \mathrm{M} \mathrm{H}_{2} \mathrm{O}$ \\
Gravity & $-9.87 \mathrm{~m} / \mathrm{s}^{2}$ (in the z-direction) \\
Fluid velocities at the chamber inlet & Varied from $0.0125 \mathrm{~m} / \mathrm{s} \mathrm{to} 0.5 \mathrm{~m} / \mathrm{s}(0.29 \mathrm{ml} / \mathrm{min}$ to $11.78 \mathrm{ml} / \mathrm{min})$ \\
Velocity of soluble factor fluid at the microchannel inlets & $0.001 \mathrm{~m} / \mathrm{s}(0.024 \mathrm{ml} / \mathrm{min})$ \\
Maximum number of CFD iterations & 300 \\
CFD convergence criterion & 0.0001 \\
Minimum CFD residual & $1 \times 10^{-18}$ \\
\hline
\end{tabular}


delivered and the intensity profiles were characterized. FITC solution $(15 \mu \mathrm{M})$ was constantly pumped into the microchannel inlets using a digital syringe pump (NE-1000 Multi-Phaser ${ }^{\mathrm{TM}}$ ) at a flow rate of $0.024 \mathrm{ml} / \mathrm{min}$, corresponding to a flow velocity of $0.001 \mathrm{~m} / \mathrm{s}$; while the entering velocity of bath fluid at the chamber inlet was varied from $0.6 \mathrm{ml} / \mathrm{min}$ to $7.1 \mathrm{ml} / \mathrm{min}(0.025 \mathrm{~m} / \mathrm{s}$ to $0.3 \mathrm{~m} / \mathrm{s})$ using a digital peristaltic pump (Marlow 400 pump from Watson Marlow Bredel Inc.). Images of dye entering the chamber were acquired using fluorescence microscopy.

\subsection{Simulating mass transport through a modeled brain slice}

To assess the transport of soluble factors through a brain slice, fluid flow through a volume representing a material of porous matrix was simulated. Figure 1(b) shows a 3D finite element meshed model of the geometry that represents the fluid body of the perfusion chamber, a microchannel and a $250 \mu \mathrm{m}$ thick brain slice. For this model, one single microchannel was used to track the mixing of chemicals in a porous matrix, as fluid flow through each of the four microchannels has similar characteristics since all of them have the same width and moreover the via holes have the same diameter. The volume that mimics the brain tissue is considered an isotropic homogeneous porous medium with a non-deformable cell matrix of a constant porosity of 0.4 , a permeability of $1 \mathrm{E}-11 \mathrm{~m}^{2}$, and a tortuosity of 1.5 , all based on previously reported values (Kalyanasundaram et al. 1997).

\subsection{Experimental study for assessing transport through a brain slice}

To evaluate the accuracy of CFD simulations that included a simulated brain slice, mass transport through an actual $250 \mu \mathrm{m}$ thick brain slice under the corresponding actual conditions was carried out. CD1 mice were anesthetized with AErrane (isoflurane, USP) and decerebrated. All procedures were approved by the Animal Care and Use Committee at the University of Illinois at Chicago. The frontal lobe was isolated in ice-cold standard solution and glued onto a tissue base. After slicing the tissue across the frontal lobe of the brain with a tissue slicer (VT1000S, Leica Microsystems Inc.) into 250-350 $\mu \mathrm{m}$ thick sections, the brain slices were incubated in artificial cerebral spinal fluid (ACSF) standard solution $(73.04 \mathrm{~g} \mathrm{NaCl}, 21.84 \mathrm{~g}$ $\mathrm{NaHCO} 3,55.74$ g glucose, 3.36 g NaH2 PO4 7H2O, $1.87 \mathrm{~g} \mathrm{KCl}, 1,000 \mathrm{ml}$ of DI water, $2.4 \mathrm{ml}$ of $\mathrm{CaCl} 2$ and $1.2 \mathrm{ml}$ of $\mathrm{MgCl} 2$ ) which was continuously aspirated with $95 \% \mathrm{O} 2-5 \% \mathrm{CO} 2$ and maintained at $34^{\circ} \mathrm{C}$ (for the initial $1 \mathrm{~h}$ and later at room temperature) before using them in experiments.
FITC dye solution was used again as a model soluble factor and the intensity was quantified with time-lapse fluorescence microscopy. FITC dye solution $(15 \mu \mathrm{M})$ dispensed at one inlet port was constantly pumped into the microchannel at a flow rate of $0.024 \mathrm{ml} / \mathrm{min}(0.001 \mathrm{~m} / \mathrm{s})$ using a digital peristaltic pump (NE-1000 Multi-PhaserTM) and driven into the brain slice through the via, finally emerging into the perfusion chamber bath at the topside of the slice. MATLAB ${ }^{\circledR}$ was used for image processing. A rectangular region centrally located over the fluorescent spot was selected; then within that region ten lines, each one separated ten intensity points from one another, were chosen to generate one single line after vertically averaging the intensity points of each of the ten lines. Finally, the Curve Fitting Toolbox of Matlab was used to run the Moving Average Filter (span=9). This filter is well known when dealing with measurement noise.

\section{Results and discussion}

\subsection{Comparing flow simulations with fluorescence microscopy observations}

In order to evaluate the accuracy of CFD simulations, simulations of the flow through the $\mu \mathrm{BSD}$ with chamber inlet volumetric flow rates ranging from $0.6 \mathrm{ml} / \mathrm{min}$ to $5.8 \mathrm{ml} / \mathrm{min}(0.025 \mathrm{~m} / \mathrm{s}$ to $0.25 \mathrm{~m} / \mathrm{s})$ at the chamber inlet were compared to fluorescence microscopy observations of the actual flow through the $\mu \mathrm{BSD}$ under the same conditions. It is important to mention that the actual flow through the device is modeled as fluid-wall interfaces. Fluid-air interfaces are not included in this study as exist at the top of the open bath perfusion chamber. However, this minimal restriction of model predicts experimental fluid flow accurately as the simulations were found to correctly predict experimental flow behavior, particularly in the vicinity of the inlets, outlets, and via holes.

Close attention was also given to the flow patterns of the fluorescent dye injected into the device to validate the simulation as shown in Fig. 2(c), (f), and (i). At the steady state conditions, the dye stream would emerge from the via holes in a cloud-like nature. This flow pattern matched the flow behavior described by the molar concentration profiles of the simulations as shown in Fig. 2(b), (e), and (h).

\subsection{Tracking the mixing of chemicals in a porous matrix}

The objective of this particular simulation was to predict the distribution of a drug or chemical in a homogenous region of the brain by using computational fluid analysis. We wished to assess the effect that a $250 \mu \mathrm{m}$ wide channel with a $65 \mu \mathrm{m}$ wide via hole have on the spatial intensity 
(a)
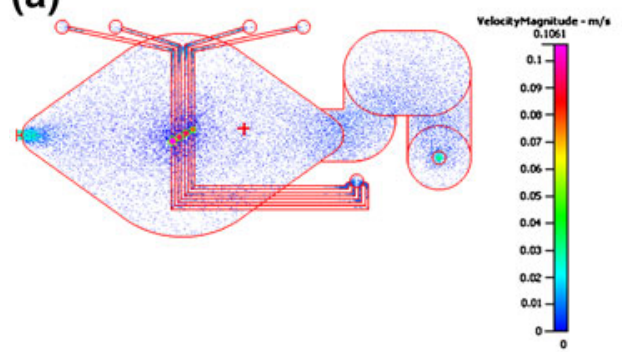

(d)
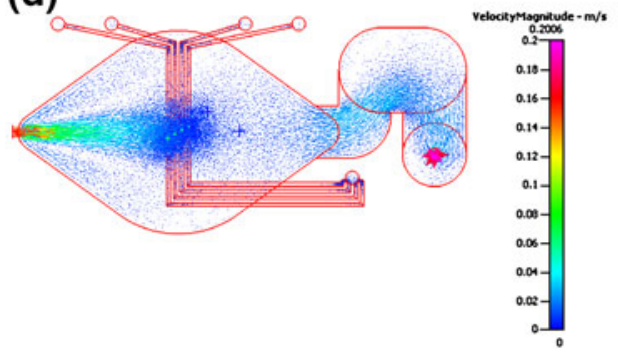

(g)

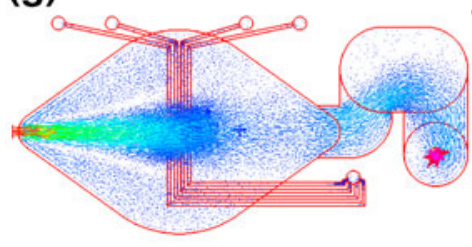

(b)
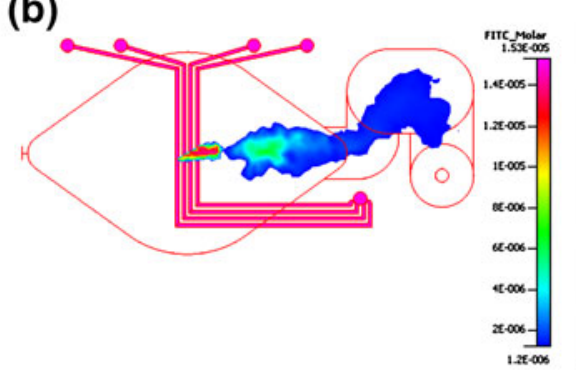

(c)

(e)
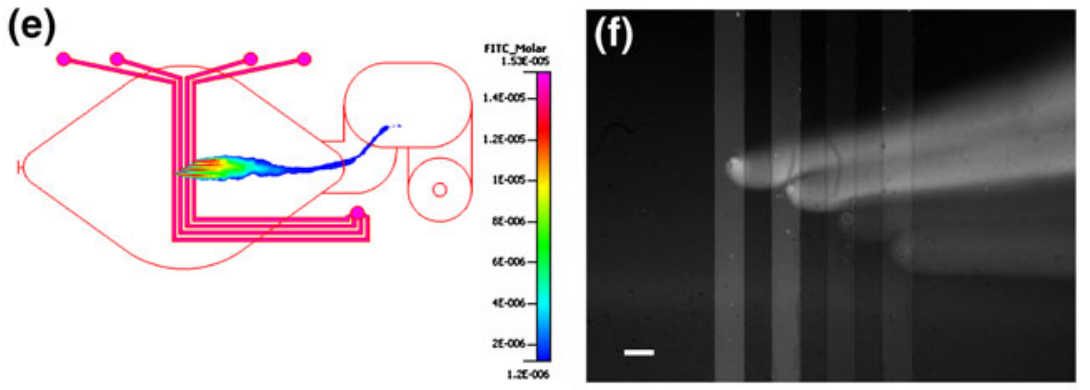

(h)

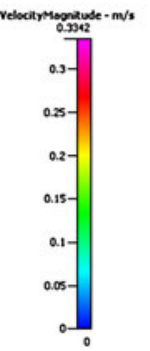

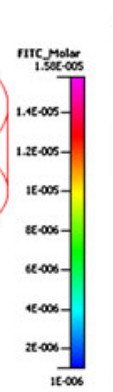

(i)

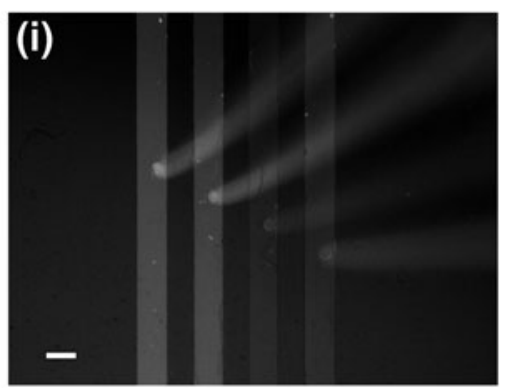

Fig. 2 Fluid velocity profiles (a, d, g), FITC molar concentration profiles (b, e, h) and photographs of FITC solution pumped into the chamber through the via holes $(\mathbf{c}, \mathbf{f}, \mathbf{i})$. From top figures to bottom figures, entering volumetric flow rate, $\mathrm{Q}$, of bath fluid at the chamber inlet increases. For figures $(\mathbf{a}-\mathbf{c}), Q=0.60 \mathrm{ml} / \mathrm{min}$ (corresponding to entering fluid velocity $v=0.025 \mathrm{~m} / \mathrm{s}$ ); FITC solution emerges from the via holes in a cloud-like nature. For figures $(\mathbf{d}-\mathbf{f}), Q=3.5 \mathrm{ml} / \mathrm{min}$ $(v=0.150 \mathrm{~m} / \mathrm{s})$; a jet stream is observed in the bulk fluid. For figures (g-i), $Q=5.8 \mathrm{ml} / \mathrm{min}(v=0.250 \mathrm{~m} / \mathrm{s})$; the influence of the jet stream is even more apparent. The jet stream develops with increasing fluid velocity at the chamber inlet. In turn, the jet stream increases chamber pressures, decreasing the ability of FITC solution to enter the chamber through the via holes. In all figures, the scale bar measures $250 \mu \mathrm{m}$

species distribution was expected in the region where the dyesoluble factor fluid was dispensed. The simulation confirmed the expectation by resulting in a symmetrical bell-shaped species distribution with a maximum width of approximately $400 \mu \mathrm{m}$ that corresponds to about three standard deviations from the origin, which agrees with actual experimental results found in the same time frame (Fig. 3(c)).

It should also be known that our current model of a brain slice contains simplifying assumptions. It does not take into account poroelastic deformation tissue behavior, which could have an effect on the transport of drugs or other soluble factors delivered to the brain tissue. Our model should be considered as a method for assessing the mixing of chemicals and water based solutions through a material that is mimicked by a simulated volume with some of the approximated properties of brain tissue (porosity, permeability and tortuosity).
Since the volume that mimics the brain tissue was specified as an isotropic, homogeneous, porous medium; a symmetrical 


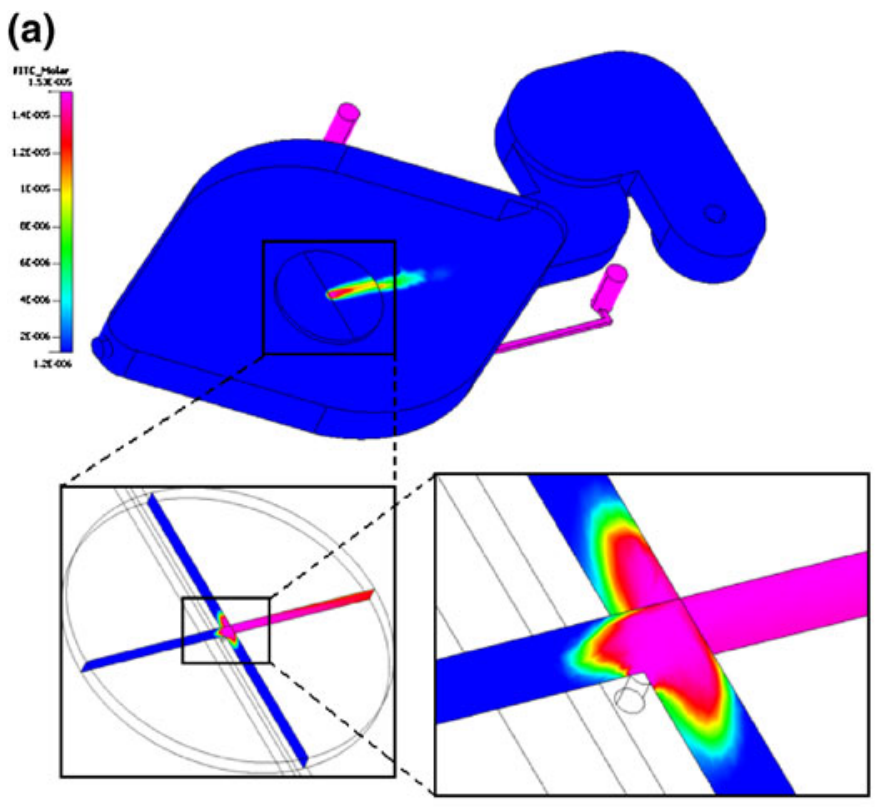

(b)

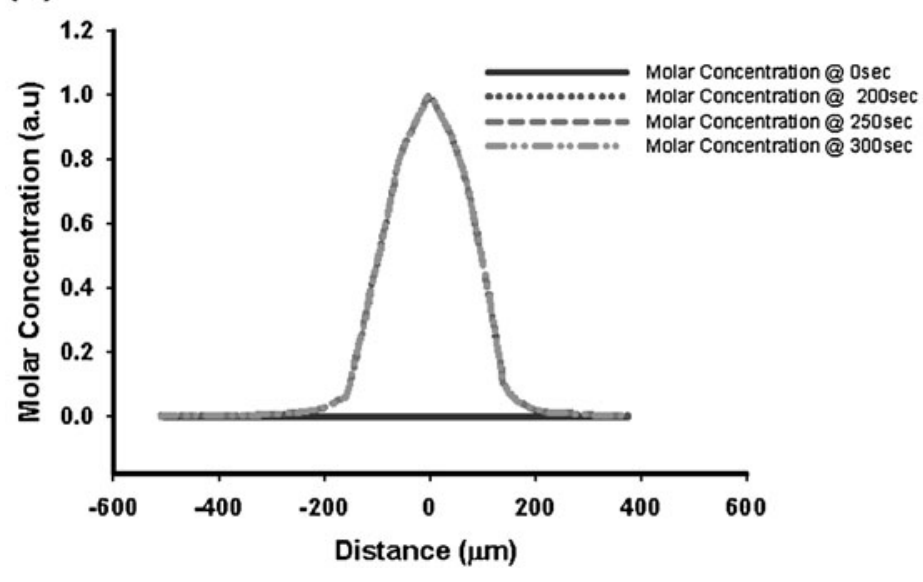

Fig. 3 Spatial profiles for assessing transport through porous media. (a) Fluid body of the $\mu \mathrm{BSD}$ including a porous central disc. (b) Spatial FITC molar concentration profile results of the simulations with the model shown in (a) at $t=0,200 \mathrm{~s}, 250 \mathrm{~s}$, and $300 \mathrm{~s}$ after

\subsection{Spatial intensities profiles for assessing transport through a brain slice}

Figure 3(c) shows FITC fluorescence intensities at different times $(0 \mathrm{~s}, 200 \mathrm{~s}, 250 \mathrm{~s}$, and $300 \mathrm{~s}$ after continuous injection of FITC at an inlet port) along the diameter of a single via (65 $\mu \mathrm{m}$ wide) covered by a brain slice and at different distances from the center of the via. As is evident from the constant final intensities and lack of broadening of the fluorescence intensity profiles, mass transport of the FITC dye solution through the brain slice and through the ACSF solution of the bulk bath occurs at a constant rate. From Fig. 3(c) it is clear that the dye is driven through the brain slice and finally into the bulk ACSF by forced convection as a result of constant pumping through the porous structure

\section{(c)}

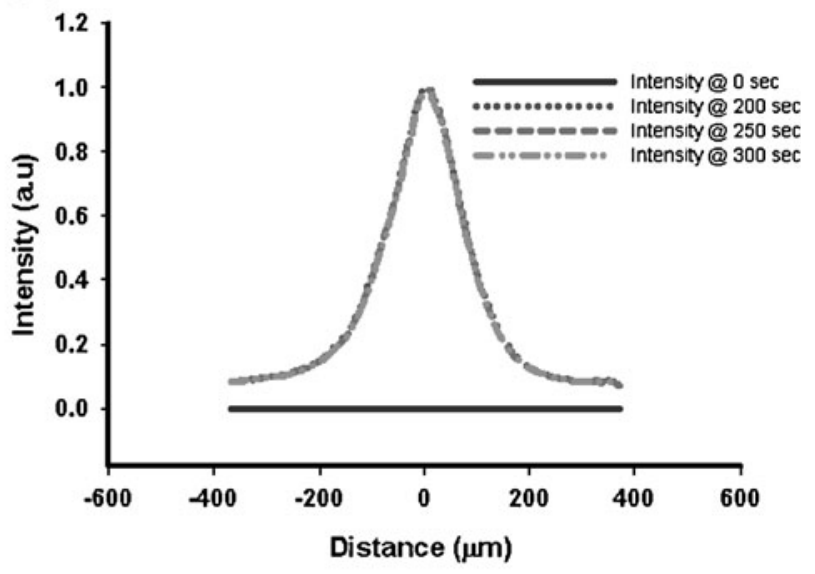

continual simulation. (c) Experimental results of the spatial FITC fluorescence intensity profiles in a brain slice tissue at $t=0 \mathrm{~s}, 200 \mathrm{~s}$, $250 \mathrm{~s}$, and $300 \mathrm{~s}$ after continual FITC dispension at an inlet port

of the slice. In contrast, if the transport was diffusion driven, the resulting fluorescence curve would be expected to broaden while the baseline would rise with time and the peak value would remain unchanged. In previous work (Mohammed et al. 2008) we obtained similar convection driven results by using transient passive pumping as a driving force. The difference in those profiles is that they were not as smooth as with constant pumping, narrowing rapidly around the peaks after reaching their maximum intensities. Therefore, passive pumping method provides precise control over fluid delivery and eliminates the need for tubing, connectors, and pumps. However, if longer stimulating time windows are required, then multiple passive pumping injections of the same chemical compound are difficult without adapting complex pulsing 
methods (Resto et al. 2010) and constant fluid pumping with standard perfusion setups may prove simpler.

\section{Conclusion}

Modeling of the flow of fluids through a $\mu$ BSD and through a volume that mimics a brain slice was conducted with CFD-ACE + multi-physics software. The simulations obtained from these methods accurately predicted the fluorescence microscopy observations of the flow of liquid chemicals through a $\mu \mathrm{BSD}$. Therefore, the simulation results obtained from this study provide a reliable tool for understanding the flow of bath and delivered microfluidic flows through the $\mu \mathrm{BSD}$ and for controlling the in vitro microenvironment that ensues during operation. Furthermore, although our current model of a brain slice adopts several assumptions to simplify the analysis, the simulation data is found to be in good enough agreement with actual experimental results to be useful for physiology research.

Acknowledgements Funding was provided by NIH MH-085073 (CPF, DTE), NIH MH-64611 (CPF), and a NARSAD Young Investigator Award (CPF).

Open Access This article is distributed under the terms of the Creative Commons Attribution Noncommercial License which permits any noncommercial use, distribution, and reproduction in any medium, provided the original author(s) and source are credited.

\section{References}

G.K. Aghajanian, K. Rasmussen, Intracellular studies in the facial nucleus illustrating a simple new method for obtaining viable motoneurons in adult-rat brain-slices. Synapse 3(4), 331-338 (1989)

E. Berthier, D.J. Beebe, Flow rate analysis of a surface tension driven passive micropump. Lab Chip 7(11), 1475-1478 (2007)
A.J. Blake et al., Multilayer PDMS microfluidic chamber for controlling brain slice microenvironment. Lab Chip 7(7), 842849 (2007)

Y. Choi et al., Three dimensional MEMS microfluidic perfusion system for thick brain slice cultures. Biomed Microdevices 9(1), 7-13 (2007)

T.L. Crowder et al., Kainate receptor activation potentiates GABAergic synaptic transmission in the nucleus accumbens core. Brain Res 1088(1), 73-82 (2006)

R. Dingledine et al., The in vitro brain slice as a useful neurophysiological preparation for intracellular-recording. J Neurosci Meth 2(4), 323-362 (1980)

ESI CFD Research Corporation, CFD-ACE+, user's manual, V2009.0 (Huntsville, AL, 2009)

B.H. Gahwiler, Organotypic monolayer cultures of nervous tissue. J Neurosci Meth 4(4), 329-342 (1981)

B.H. Gahwiler, Organotypic cultures of neural tissue. Trends Neurosci 11(11), 484-489 (1988)

H.L. Haas et al., A simple perfusion chamber for the study of nervous tissue slices in vitro. J Neurosci Meth 1(4), 323-325 (1979)

S. Kalyanasundaram et al., A finite element model for predicting the distribution of drugs delivered intracranially to the brain. Am J Physiol 273(5 Pt 2), R1810-R1821 (1997)

S.R. Kelso et al., A slice chamber for intracellular and extracellular recording during continuous perfusion. Brain Res Bull 10(6), 853-857 (1983)

A.A. Linninger et al., Prediction of convection-enhanced drug delivery to the human brain. J Theor Biol 250(1), 125-138 (2008)

M. Mameli et al., Developmentally regulated actions of alcohol on hippocampal glutamatergic transmission. J Neurosci 25(35), 8027-8036 (2005)

J.S. Mohammed et al., Microfluidic add-on for standard electrophysiology chambers. Lab Chip 8(7), 1048-1055 (2008)

K.B. Neeves et al., Fabrication and characterization of microfluidic probes for convection enhanced drug delivery. J Control Release 111(3), 252-262 (2006)

P.A. Passeraub et al., Design, microfabrication and analysis of a microfluidic chamber for the perfusion of brain tissue slices. Biomed Microdevices 5(2), 147-155 (2003)

P.J. Resto et al., An automated microdroplet passive pumping platform for high-speed and packeted microfluidic flow applications. Lab Chip 10(1), 23-26 (2010)

G.M. Walker et al., Microenvironment design considerations for cellular scale studies. Lab Chip 4(2), 91-97 (2004) 